Introduction Changes in grey matter volume (GMV) and cortical thickness (CT) have been previously documented in adult Crohn's Disease (CD) patients in remission, however data is inconsistent and not explored in patients with active CD. Here, we investigate alterations in GMV and CT in patients with active $\mathrm{CD}$, and their correlation with disease activity biomarkers such as faecal calprotectin (FCP), C-reactive protein (CRP), Interleukin-6 (IL-6), Interleukin-1 $\beta$ (IL-1 $\beta$ ) and TNF $\alpha$ Methods 25 active ileal/colonic CD patients and 25 healthy controls (HC) age-, BMI- and gender-matched were scanned. Active CD was defined as Harvey Bradshaw index $>5$ and CRP $>5 \mathrm{mg} / \mathrm{dl}$, or FCP $>250 \mu \mathrm{g} / \mathrm{g}$ or assessed through ileocolonoscopy or magnetic resonance enterography. Cytokine levels were measured from serum samples using colorimetric ELISA. FCP were acquired at inclusion. Anatomical T1-weighted brain images were acquired on a $3 \mathrm{~T}$ Philips Achieva scanner using a 32-channel receive coil. GMV was measured using voxel based morphometry and cortical thickness was determined using computational anatomy toolbox (CAT12) in Statistical Parametric Mapping (SPM) software.

Results In CD patients, significant cortical thinning in the right rostral middle-frontal area (involved in attention and working memory) was found compared with HCs. A significant decrease in GMV was found in CD compared with $\mathrm{HC}$ in pre-central gyrus (motor cortex) and post-central gyrus (somatosensory cortex). Correlating IL-6 with CT in CD showed cortical thinning in the right superior-frontal area (involved in self-awareness) and right lingual regions (involved in vision processing) with increasing IL-6 levels. IL-6 was negatively correlated with the GMV in bilateral insula (involved in pain processing) and pre- and post-central gyrus. FCP was negatively correlated with the right insula. Further cytokine assays of TNF $\alpha$ and IL-1 $\beta$ are presently underway in this study.

Conclusions A significant reduction in CT and GMV in cortical areas has been shown in patients with active CD compared with HCs. This observation may be associated with a chronic inflammatory response, attributed to high levels of circulating neurotoxic cytokines. Serum TNF $\alpha$ and IL-1 $\beta$ levels will also be correlated to CT and GMV in this study to validate the aforementioned observation. Investigating structural brain changes in active CD will aid our understanding of the crosslinking between chronic inflammation, brain morphology and changes in behaviour, cognitive function and unexplained symptoms such as fatigue in CD. This will inform new medical and psychological therapies.

\section{PWE-064 COMBINED INFLAMMATORY BOWEL DISEASE- OBSTETRIC CLINIC, A DISTRICT GENERAL HOSPITAL EXPERIENCE OVER 2 YEARS}

Nora Thoua*, Alexandra Hall, Cinzia Papadia, Edgar Dorman, Maryam Parisaei. Homerton University Hospital, London, UK

\subsection{6/gutjnl-2018-BSGAbstracts. 196}

Background Management of pregnant women with inflammatory bowel disease (IBD) can be complex. Women often report getting conflicted information from different health care professionals and needing to attend too many hospital appointments. Following the success of other combined medical-obstetric clinics that were already running at Homerton hospital we set up a monthly IBD - obstetric clinic in January
2016. The aim of this study was to review the effect of this clinic on pregnancy outcomes.

Method A retrospective review of patient records was performed to obtain patient demographics, medical, surgical and drug history, mode of delivery and birth weight from January 2016 to January 2018.

Results A total of 45 pregnancies in 44 women were identified. 18 women have Crohn's disease (CD) and 26 ulcerative colitis (UC). Most women were on some treatment with only 4 being on none. 21 women were on 5-ASA (oral, topical or both). 7 women were on thiopurines. 8 women were on biologics (Infliximab 3, adalimumab 4, vedolizumab 1). 5 women were on biologics and thiopurines ( 3 adalimumab, 2 infliximab). Biologics were stopped at 28 weeks in $6 / 8$ women, 1 woman stopped at 20 weeks and in one case it was necessary to continue Adalimumab throughout the pregnancy.

2 patients needed treatment with prednisolone due to flare up during pregnancy. One woman was diagnosed with UC during pregnancy and required prednisolone. One woman with severe perianal CD needed surgical drainage during pregnancy.

All pregnancies resulted in live births. Mean birth weight was 3203 g. 7 women had emergency caesarean section (CS), 9 women elective CS and 5 had instrumental deliveries. The commonest indications for elective CS were obstetric or maternal choice and emergency CS foetal distress or failure to progress. There were 5 preterm deliveries ( $<37$ weeks), 4 spontaneous, 1 emergency. There was one birth with severe intrauterine growth retardation (IUGR) secondary to a large placental haemorrhage at the beginning of the pregnancy and 1 duodenal atresia.

One woman on infliximab and azathioprine developed listeria sepsis 10 days after the last infliximab infusion at 28 weeks. This was identified and treated appropriately, the pregnancy continued to term with no foetal complications.

Average number of clinic visits was 3 . There were a total of 115 appointments with a rising trend as the clinic became established and better known to GPs and midwives.

Conclusion This study showed that a combined IBD-obstetric clinic improves adherence to treatment and guidelines with good pregnancy outcomes. Patient feedback is that they value this combined approach both in terms of the medical/obstetric expertise and in terms of convenience.

\section{PWE-065 ANTIBODIES-TO-INFLIXIMAB POST INDUCTION CAN HELP IDENTIFY INFLAMMATORY BOWEL DISEASE PATIENTS AT RISK OF TREATMENT FAILURE}

Gloria Shwe Zin Tun*, Sister Laura Marshall, Sister Kerry Robinson, Sister Alison Wright, Dr Alenka Brookes, Thean S Chew, Melissa Hale, Alan J Lobo. Sheffield Teaching Hospitals NHS Foundation Trust, Sheffield, UK

\subsection{6/gutjnl-2018-BSGAbstracts. 197}

Introduction Therapeutic drug monitoring (TDM) of infliximab (IFX) aids decision-making in patients with inflammatory bowel disease (IBD) since subtherapeutic IFX levels and the formation of antibodies to infliximab (ATI) are associated with treatment failure. However, the optimal time to perform TDM remains unclear and practice varies.

Aim To assess prevalence of positive ATI after induction therapy, risk factors for their development and relationship to treatment outcome. 